\title{
Management of New Post-Operative Arrhythmia in Cardiac Patients
}

\author{
Michael MacPherson", Gwyn Beattie, Robyn Smith, John Butler \\ From World Society of Cardiothoracic Surgeons 25th Anniversary Congress, Edinburgh \\ Edinburgh, UK. 19-22 September 2015
}

\section{Background/Introduction}

Literature suggests $25-50 \%$ of patients develop arrhythmia following cardiac surgery. Within a regional cardiothoracic centre in Scotland, guidelines exist on initial management of post-operative atrial arrhythmia. However, in practice various approaches are used. Arrhythmias contribute to prolonged hospital stay and high dependency readmission.

\section{Aims/Objectives}

To assess staff awareness of local guidelines, audit the proportion of patients developing arrhythmia and check for correlation with logistic EuroScore and length of hospital stay. Approach to management of arrhythmia within the initial 48 hours following identification was compared to local guidelines.

\section{Method}

Staff questionnaire was circulated prior to a four week data collection period. Consecutive cases of new post-operative arrhythmia were identified within the intensive care unit, high dependency unit and cardiac ward. Contributions were made to the proforma by anaesthetic, cardiology and surgical consultants.

\section{Results}

Results from the staff questionnaire indicated $64 \%$ were unaware of local guidelines. 25 cases were identified; 2 in ICU, 15 in HDU and 8 on the ward. Case mix included CABG (37\%), valve replacement (20\%), mixed CABG and valve (23\%), and other (20\%). Average logistic EuroScore was $4.34 \%$, higher than the centres average. In the majority of cases (56\%), onset of arrhythmia occurred 24-72 hours following surgery. Arrhythmia most frequently identified was atrial fibrillation (22 cases). There was one case each of SVT, NSVT and atrial flutter. During analysis of atrial fibrillation cases, when asking 'would management have differed if local guidelines were adhered to?', the answer was yes in $8(36 \%)$ of cases. In the majority of cases arrhythmia lasted less than 48 hours. 2 cases were cardioverted. Duration of inpatient stay for this study group was 9.6 days, significantly longer than the centre's average of 6 days.

\section{Discussion/Conclusion}

There is not a definite treatment strategy for the management of new onset atrial arrhythmias following cardiac surgery. Management most frequently included potassium and magnesium replacement, oral beta blocker and IV amiodarone. This project raises the question as to whether a standardised guideline is feasible in practice. Complexities include variation in patient suitability for management and consultant preferences.

Published: 16 December 2015

doi:10.1186/1749-8090-10-S1-A137

Cite this article as: MacPherson et al:: Management of New PostOperative Arrhythmia in Cardiac Patients. Journal of Cardiothoracic Surgery 2015 10(Suppl 1):A137. 